\title{
Time-Dependent Thermopower Effect in an Interacting Quantum Dot
}

\author{
M. Bagheri Tagani, H. Rahimpour Soleimani, \\ Department of physics, University of Guilan, P.O.Box 41335-1914, Rasht, Iran
}

June 18, 2018

\begin{abstract}
The time-dependent thermopower is analyzed through an interacting quantum dot coupled to a time-dependent gate voltage and under the influence of an external magnetic field using the Keldysh nonequilibrium Green's function formalism. Formal expressions of the electrical and thermal conductances, thermopower, and thermoelectrical figure of merit are obtained. The influence of the magnetic field on the displacement current and the heat current is studied. Results show that although applying time-dependent gate voltage results in the enhancement of the Seebeck coefficient, the electron-electron interaction gives rise to a significant reduction in the thermopower. The reason for why applying time dependent gate voltage results in the enhancement of the thermopower is also analyzed.
\end{abstract}

\section{Introduction}

Thermopower - the ratio of induced voltage to an applied temperature gradient across the sample at the state of vanishing current - is one of the oldest issues of solid-state physics. The study of thermopower in nanoscale devices has attracted a lot of attention in recent years because of recent developments in fabricating and utilizing them. In addition, deviations from the Wiedmann-Franz law in nanostructures result in significant enhancements in the thermopower of such devices [1. Various models have been suggested for the study of thermoelectric transport through quantum dots (QDs) and molecular junctions [2, 3, 4, 5, 6, 7, nanotubes and nanowires [8, 9, 10], strongly correlated nanostructures [11, 12, [13, etc. In addition, measurement of the thermopower through nanostructures has been an interesting topic in recent years [14, 15, 16, 17, 18.

Recently, Crepieux and co-workers [19] proposed applying time-dependent gate voltage results in the enhancement of the thermopower. Their results showed that the thermopower is enhanced by up to $40 \%$. Time-dependent transport has been extensively studied both theoretically and experimentally 20, 21, 22, 23, 24, 25, 26, 27. However, the time-dependent heat current and 
the thermopower are novel phenomena requiring more attention. In this paper, we consider an interacting QD coupled to metal leads. Although the system seems to be similar to what was considered in Ref. [19], the effects of electronelectron interactions and the magnetic field are also taken into account. Using the Keldysh nonequilibrium Green's function formalism [28, 29], expressions for the electrical and thermal conductance are obtained. The results show that the time-dependent thermopower is significantly reduced by electron-electron interactions. The influence of the magnetic field on the heat current is also investigated.

In the next section, the heat current is evaluated using the nonequilibrium Green's function formalism. We use the Hartree approximation and hence the heat current is related to the averaged electron density. In sect. 3, numerical results are presented and in the end, conclusion is presented.

\section{Model}

We consider an interacting QD coupled to metal leads and under the influence of a step-like gate voltage pulse. The Hamiltonian describing the system is given as follows:

$$
H=\sum_{\alpha k \sigma} \varepsilon_{\alpha k \sigma} c_{\alpha k \sigma}^{\dagger} c_{\alpha k \sigma}+\sum_{\sigma} \varepsilon_{\sigma}(t) n_{\sigma}+U n_{\uparrow} n_{\downarrow}+\sum_{\alpha k \sigma}\left[V_{\alpha k \sigma} c_{\alpha k \sigma}^{\dagger} d_{\sigma}+H . C\right]
$$

where $c_{\alpha k \sigma}\left(c_{\alpha k \sigma}^{\dagger}\right)$ destroys (creates) an electron with wave vector $k$, spin $\sigma$, and energy $\varepsilon_{\alpha k \sigma}$ in lead $\alpha(\alpha=\mathrm{L}$ or $\mathrm{R}) . d_{\sigma}\left(d_{\sigma}^{\dagger}\right)$ is the annihilation (creation) operator for the dot and $n_{\sigma}=d_{\sigma}^{\dagger} d_{\sigma}$ is the occupation number. $\varepsilon_{\sigma}(t)$ denotes the time-dependent energy level of the QD defined as $\varepsilon_{\sigma}(t)=\varepsilon_{\sigma}^{0}+\Delta_{d} \Theta(t)$ where $\Delta_{d}$ is the time-variation of the gate voltage. $\varepsilon_{\sigma}^{0}=\varepsilon_{0} \pm E_{z}$ (plus sign for spin-up) is the time-independent energy level of the QD and $E_{z}$ is Zeeman splitting induced by an external magnetic field. $U$ and $V_{\alpha k \sigma}$ stand for Coulomb repulsion and tunneling strength between the dot and the lead $\alpha$, respectively. The time-dependent heat current $\left(I^{\mathrm{h}}\right)$ is obtained from the difference between the energy current $\left(I^{\mathrm{e}}\right)$ and the charge current $\left(I^{\mathrm{q}}\right)$ according to

$$
I_{\alpha}^{\mathrm{h}}(t)=I_{\alpha}^{\mathrm{e}}(t)-\frac{\mu_{\alpha}(t)}{e} I_{\alpha}^{\mathrm{q}}(t)
$$

where $I_{\alpha}^{\mathrm{q}}=-\sum_{k \sigma \in \alpha}<\frac{\mathrm{d}}{\mathrm{dt}} c_{\alpha k \sigma}^{\dagger} c_{\alpha k \sigma}>, I_{\alpha}^{\mathrm{e}}=-\sum_{k \sigma \in \alpha} \varepsilon_{\alpha k \sigma}<\frac{\mathrm{d}}{\mathrm{dt}} c_{\alpha k \sigma}^{\dagger} c_{\alpha k \sigma}>$, and $\mu_{\alpha}$ denotes the chemical potential of lead $\alpha$. The Keldysh nonequilibrium Green's function formalism is used to obtain the energy current. It is straightforward to show that by means of the Green's function of the isolated leads, the energy current is given as $(\hbar=1)$ [19]

$$
\begin{gathered}
I_{\alpha}^{\mathrm{e}}(t)=2 R e \sum_{k \sigma \in \alpha}\left|\mathrm{V}_{\alpha k \sigma}\right|^{2} \int_{-\infty}^{t} \mathrm{~d} t_{1} \mathrm{i} \varepsilon_{\alpha k \sigma} \mathrm{e}^{-\mathrm{i} \varepsilon_{\alpha k \sigma}\left(t_{1}-t\right)} \\
{\left[f_{\alpha}\left(\varepsilon_{\alpha k \sigma}\right) G_{\sigma \sigma}^{\mathrm{r}}\left(t, t_{1}\right)+G_{\sigma \sigma}^{<}\left(t, t_{1}\right)\right]}
\end{gathered}
$$


where $f_{\alpha}(\varepsilon)=\left[1+\exp \left(\left(\varepsilon-\mu_{\alpha}\right) / k T_{\alpha}\right)\right]^{-1}$ is the Fermi distribution function of the $\alpha^{t h}$ lead and $T_{\alpha}$ denotes the temperature of the lead. $G_{\sigma \sigma^{\prime}}^{r}\left(t, t^{\prime}\right)$ and $G_{\sigma \sigma^{\prime}}^{<}\left(t, t^{\prime}\right)$ are the retarded and the lesser Green's functions of the interacting $\mathrm{QD}$, respectively.

The Green's function of the QD is obtained from a Dayson-like equation as

$$
G_{\sigma \sigma^{\prime}}\left(\tau, \tau^{\prime}\right)=g_{\sigma \sigma^{\prime}}\left(\tau, \tau^{\prime}\right)+\int_{C} \mathrm{~d} t_{1} \mathrm{~d} t_{2} g_{\sigma \sigma^{\prime}}\left(\tau, \tau_{1}\right) \Sigma_{\sigma}\left(\tau_{1}, \tau_{2}\right) G_{\sigma \sigma^{\prime}}\left(\tau_{2}, \tau\right)
$$

where $C$ denotes the contour integral, and $\Sigma_{\sigma}\left(t_{1}, t_{2}\right)=\sum_{\alpha k}\left|V_{\alpha k \sigma}\right|^{2} g_{\alpha k \sigma}\left(t_{1}, t_{2}\right)$ is the self-energy operator in which $g_{\alpha k \sigma}\left(t, t^{\prime}\right)$ is the isolated lead Green's function. $g_{\sigma \sigma^{\prime}}$ is the isolated QD Green's function satisfying 30]

$$
\left[\mathrm{i} \frac{\mathrm{d}}{\mathrm{d} t}-\varepsilon_{\sigma}(t)-U<n_{\bar{\sigma}}(t)>\right] g_{\sigma \sigma^{\prime}}\left(t, t^{\prime}\right)=\delta\left(t-t^{\prime}\right) \delta_{\sigma \sigma^{\prime}}
$$

For deriving the above equation, the decoupling approximation introduced in Ref. [21] has been used, i.e., $<\left\{n_{\bar{\sigma}}(t) d_{\sigma}(t), d_{\sigma^{\prime}}^{\dagger}\left(t^{\prime}\right)\right\}>\approx<n_{\bar{\sigma}}(t)>g_{\sigma \sigma^{\prime}}\left(t, t^{\prime}\right)$. $<$ $n_{\sigma}(t)>$ denotes the time averaged electron density with spin $\sigma$, and $\bar{\sigma}$ is opposite of $\sigma$. This approximation is reasonable under conditions that the temperature is low enough (lower than the level spacing) and the bias voltage is small. In the following, we change the sum over $k$ into an energy integral and use the wide band approximation, i.e., $\sum_{k}\left|V_{\alpha k \sigma}\right|^{2}=\int d \varepsilon / 2 \pi \Gamma_{\alpha}^{\sigma}$ where $\Gamma_{\alpha}^{\sigma}=2 \pi \rho_{\alpha}\left|V_{\alpha k \sigma}\right|^{2}$ is the spin-dependent tunneling rate. The retarded and lesser Green functions are obtained from Eq. 4 by means of the Langreth continuation theorem [31. Then, the time-dependent heat current is given as

$$
\begin{aligned}
I_{\alpha}^{\mathrm{h}}(t) & =-\sum_{\sigma} \Gamma_{\alpha}^{\sigma}\left[\sum_{\alpha^{\prime}} \Gamma_{\alpha^{\prime}}^{\sigma} \int \frac{\mathrm{d} \varepsilon}{2 \pi}\left(\varepsilon-\mu_{\alpha}\right) f_{\alpha^{\prime}}(\varepsilon)\left|A_{\alpha^{\prime}}^{\sigma}(\varepsilon, t)\right|^{2}\right. \\
& \left.+\int \frac{\mathrm{d} \varepsilon}{\pi}\left(\varepsilon-\mu_{\alpha}\right) f_{\alpha}(\varepsilon) \operatorname{Im}\left\{A_{\alpha}^{\sigma}(\varepsilon, t)\right\}\right]
\end{aligned}
$$

where $A_{\alpha}^{\sigma}(\varepsilon, t)$ is given by 20 ]

$$
A_{\alpha}^{\sigma}(\varepsilon, t)=\frac{\left[\varepsilon-\varepsilon_{n \sigma}+\mathrm{i} / 2 \Gamma^{\sigma}\right]-\Delta_{d} \mathrm{e}^{\mathrm{i}\left[\varepsilon-\varepsilon_{n \sigma}-\Delta_{d}+\mathrm{i} / 2 \Gamma^{\sigma}\right] t}}{\left[\varepsilon-\varepsilon_{n \sigma}+\mathrm{i} / 2 \Gamma^{\sigma}\right]\left[\varepsilon-\varepsilon_{n \sigma}-\Delta_{d}+\mathrm{i} / 2 \Gamma^{\sigma}\right]}
$$

where $\varepsilon_{n \sigma}=\varepsilon_{\sigma}+U<n_{\bar{\sigma}}>$ and $\Gamma^{\sigma}=\Gamma_{L}^{\sigma}+\Gamma_{R}^{\sigma}$. It is straightforward to show the time averaged electron density is obtained from 20

$$
\begin{aligned}
<n_{\sigma}(t)> & =\lim _{T \rightarrow \infty} \frac{1}{2 T} \sum_{\alpha} \Gamma_{\alpha}^{\sigma} \int \frac{\mathrm{d} \varepsilon}{2 \pi} f_{\alpha}(\varepsilon) \int_{-T}^{T} \mathrm{~d} t\left|A_{\alpha}^{\sigma}(\varepsilon, t)\right|^{2} \\
& =\sum_{\alpha} \int \frac{\mathrm{d} \varepsilon}{2 \pi} f_{\alpha}(\varepsilon) \frac{\left(\varepsilon-\varepsilon_{n \sigma}\right)^{2}+\Delta_{d}^{2}+\left(\frac{\Gamma^{\sigma}}{2}\right)^{2}}{\left[\left(\varepsilon-\varepsilon_{n \sigma}\right)^{2}+\left(\frac{\Gamma^{\sigma}}{2}\right)^{2}\right]\left[\left(\varepsilon-\varepsilon_{n \sigma}-\Delta_{d}\right)^{2}+\left(\frac{\Gamma^{\sigma}}{2}\right)^{2}\right]}
\end{aligned}
$$

Equation 8 should be solved self-consistently to obtain the electron density. In the following, we assume that $\Gamma_{\alpha}^{\sigma}=\Gamma_{0}$ and use $\Gamma_{0}$ as the energy unit 32 and $\hbar / \Gamma_{0}$ as the time unit. 

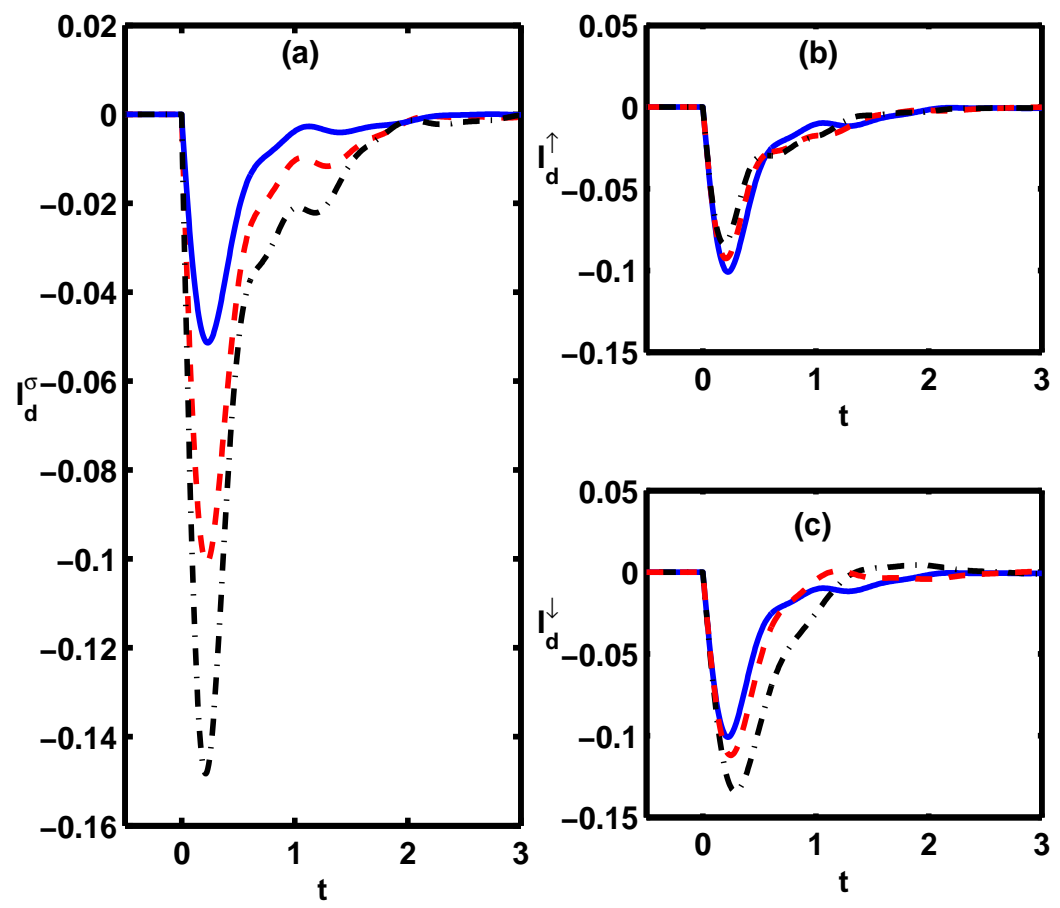

Figure 1: (a) $I_{d}^{\sigma}$ versus time for $\Delta_{d}=0.5$ (solid), $\Delta_{d}=1$ (dashed) and $\Delta_{d}=$ 1.5 (dashed-dotted). (b) and (c) show spin-resolved displacement current for $E_{z}=0$ (solid), $E_{z}=1$ (dashed) and $E_{z}=2$ (dashed-dotted). Parameters are $T_{1}=2, T_{2}=0.1, \varepsilon_{0}=0, U=2$, and $\mu_{L(R)}=+(-) 2.5$. For (b) and (c), we set $\Delta_{d}=1$. 


\section{Results and Discussion}

Figure 1 shows the spin-dependent displacement current $\left(I_{\mathrm{d}}^{\sigma}(t)=I_{\mathrm{L}}^{\sigma}(t)+I_{\mathrm{R}}^{\sigma}(t)\right)$ as a function of the amplitude of the gate voltage $\left(\Delta_{d}\right)$ (Fig. 1a) or the magnetic field (Figs. 1b and c). The displacement current describes the time evolution of the electron density of the QD. As we expect, $I_{\mathrm{d}}$ is zero at $t<0$, because the system is in the steady state. Upon applying the gate voltage, a significant reduction in $I_{\mathrm{d}}^{\sigma}$ is observed when $0<t<2$. The gate voltage shifts the QD's energy level toward the chemical potential of the emitter (left lead) and hence, a sudden decrease in the population of the charge occurs. The reduction of the population leads to $I_{\mathrm{d}}^{\sigma}<0$. During the time, the system approaches the new steady state and, therefore, $I_{\mathrm{d}}^{\sigma}$ becomes zero again. This behavior of the displacement current was recently reported in Ref. [27. Unlike the results obtained in Ref. 33], the displacement current does not show any fluctuation. Indeed, in Ref. [33 the coupling at $t=0$ adds some energy to the system but here, the gate voltage just changes the position of energy levels. Figures $1 \mathrm{~b}$ and c describe the behavior of the spin-up and spin-down displacement currents in the response to the external magnetic field, respectively. It is observed that $I_{\mathrm{d}}^{\uparrow}$ is not sensitive to the magnetic field. Indeed, in the presence of the magnetic field, the population of the spin-up is significantly reduced, because the entrance of the spin-up electron into the QD requires the energy to be more than $\mu_{\mathrm{L}}$ due to electron-electron interactions. The behavior of $I_{\mathrm{d}}^{\downarrow}$ is more interesting in the response to the magnetic field. Unlike the spin-up level, the spin-down level is always inside the bias window and the increasing magnetic field gives rise to the enhancement of the spin-down electron population. It is found that $I_{\mathrm{d}}^{\downarrow}$ becomes positive in a strong magnetic field $\left(E_{z}=2\right)$ when $1.5<t<2.5$. It comes from the inequality in the temperature of the leads.

The spin resolved heat current is plotted in Fig. 2 for different magnetic fields. The typical coherent oscillations in the heat current are observed. It is interesting to note that the frequency of the oscillations is spin- and Coulomb repulsion-dependent and given by $\hbar \omega_{\sigma}=\left|E_{f}-\varepsilon_{\sigma}-U<n_{\bar{\sigma}}>-\Delta_{\mathrm{d}}\right|$. The existence of such beats in the charge current was previously reported 22, 27; however, for the heat current more experimental results are needed. At $t<0$, or $t \rightarrow \infty$, the heat current approaches constant values. It is also observed that the variations of the heat current in the right lead are more significant than in the left one because of $T_{\mathrm{R}}<T_{\mathrm{L}}$. Notice, the heat current becomes positive when the energy level of the QD is outside the bias window. Therefore, $I_{\mathrm{L} \uparrow}^{\mathrm{h}}>0$ for $E_{z}=1$ and 2. In the case of $I_{\mathrm{L} \downarrow}^{\mathrm{h}}$, the magnitude of the heat current is enhanced by an increase of the magnetic field because of $\mu_{\mathrm{L}}>>\varepsilon_{\downarrow}$. The story is completely different about $I_{\mathrm{R} \sigma}^{\mathrm{h}}$, because an increase of magnetic field leads to $\varepsilon_{\sigma}>\mu_{\mathrm{R}}$ and hence, the magnitude of the heat current is decreased.

In the linear response limit, the current is given by $I(t)=G_{V}(t) \Delta V+$ $G_{T}(t) \Delta T$ where $G_{V}(t)$ and $G_{T}(t)$ are the electrical conductance and the thermal 

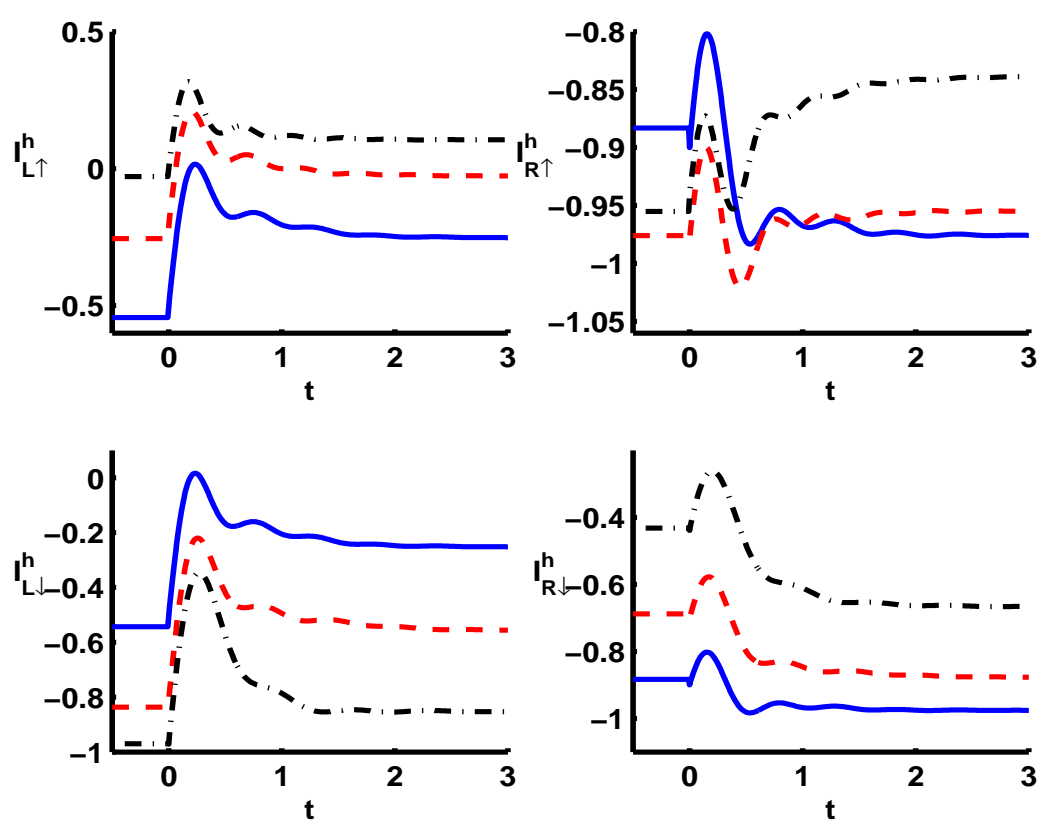

Figure 2: Spin-dependent heat current against time. $E_{z}=0$ (solid), $E_{z}=1$ (dashed) and $E_{z}=2$ (dashed-dotted). Other parameters are the same as Fig. 1.

coefficient, respectively. Setting $I(t)=0$, the Seebeck coefficient is defined as

$$
S(t)=-\frac{\Delta V}{\Delta T}=\frac{G_{T}(t)}{G_{V}(t)}
$$

From Eq. 6, we obtain the time-dependent conductance coefficients as follows 34:

$$
\begin{aligned}
& G_{V}(t)=-\sum_{\sigma} \frac{\Gamma_{\mathrm{L}}^{\sigma} \Gamma_{\mathrm{R}}^{\sigma}}{\Gamma^{\sigma}} \int \frac{\mathrm{d} \varepsilon}{\pi} f^{\prime}(\varepsilon) \operatorname{Im}\left(A^{\sigma}(\varepsilon, t)\right) \\
& G_{T}(t)=\frac{1}{T} \sum_{\sigma} \frac{\Gamma_{\mathrm{L}}^{\sigma} \Gamma_{\mathrm{R}}^{\sigma}}{\Gamma^{\sigma}} \int \frac{\mathrm{d} \varepsilon}{\pi}\left(\varepsilon-\varepsilon_{F}\right) f^{\prime}(\varepsilon) \operatorname{Im}\left(A^{\sigma}(\varepsilon, t)\right)
\end{aligned}
$$

where $f^{\prime}(\varepsilon)=\mathrm{d} / \mathrm{d} \varepsilon f(\varepsilon)$. Note that the above equations are only valid under conditions that $\Delta V$ and $\Delta T$ are small in comparison to $\varepsilon_{0}$ and $\Delta_{d}^{2} \rightarrow 0$. In these conditions, we have $\left|A^{\sigma}(\varepsilon, t)\right|^{2}=-2 / \Gamma^{\sigma} \operatorname{Im}\left[A^{\sigma}(\varepsilon, t)\right][19$. It is interesting to note that the imaginary part of $A(\varepsilon, t)$ is thought of as the generalized spectral function. The time-dependent Seebeck coefficient is plotted in Fig. 3a. One observes that there is a sudden increase in thermopower once the gate 

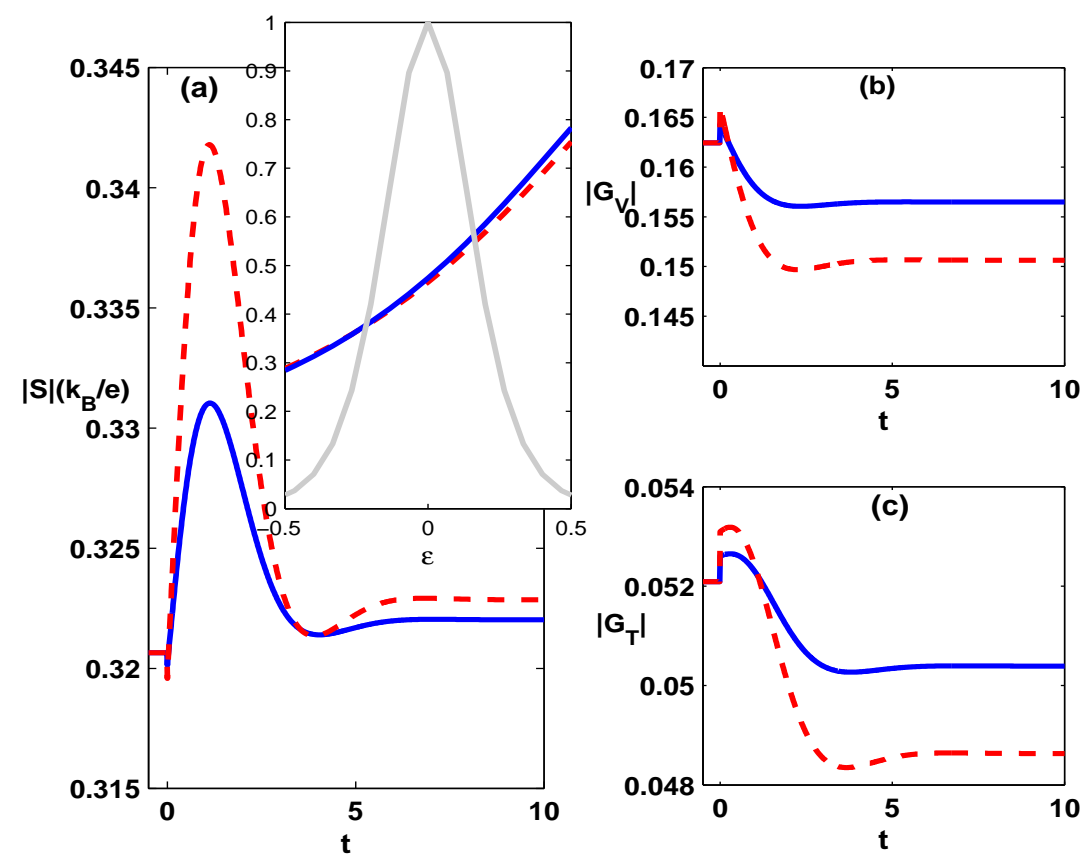

Figure 3: a) Thermopower, (b) conductance, and (c) thermal coefficient versus time. Parameters are $T_{1}=T_{2}=0.1, \varepsilon_{0}=0.5, E_{z}=0, U=2$ and $\Delta_{\mathrm{d}}=0.05$ (solid) and $\Delta_{\mathrm{d}}=0.1$ (dashed). Inset shows the generalized spectral function as a function of energy at $t=1$ (solid line) and $t=7$ (dashed line). Normalized $f^{\prime}(\varepsilon)$ is also plotted in gray.

voltage is applied. Such a time-dependent enhancement has been recently reported in a non-interacting QD 19. Indeed, the more sensitivity of the system to the temperature in the transient regime results in the enhancement of the thermopower. Since $\Delta_{\mathrm{d}}$ is small, the imaginary part of $A^{\sigma}(\varepsilon, t)$ is composed of a dominant Lorentzian-like part where $\varepsilon_{n \sigma}$ is its center, and a corrective part which is on the order of $\Delta_{\mathrm{d}}$ and decays during the time according to $\mathrm{e}^{-1 / 2 \Gamma^{\sigma} t}$. It is interesting to note that the correction part plays the main role in the enhancement of the thermopower. $f^{\prime}(\varepsilon)$ has a Lorentzian shape which is centered near the chemical potential of the lead, see inset of Fig. 3. Under these conditions, the corrective term causes $\operatorname{Im}\left(A^{\sigma}(\varepsilon, t)\right)$ to become larger at initial times after applying gate voltage. It leads to the enhancement of the thermopower. This term decays with time and, as a result, the thermopower becomes constant again. Indeed, the increasing generalized spectral function near the chemical potential of the leads results in the enhancement of the thermopower upon applying gate voltage. The generalized spectral function and $f^{\prime}(\varepsilon)$ are plotted in the inset of Fig. 3a. It is evident that the increase of $\Delta_{\mathrm{d}}$ leads to more increase 
of the generalized spectral function and, as a result, the thermopower is more enhanced.

Figs. 3b and c show the time evolution of $G_{V}$ and $G_{T}$ as a function of $\Delta_{\mathrm{d}}$, respectively. It is observed that an increase in $\Delta_{\mathrm{d}}$ leads to an increase of the variation amplitude of $G_{V}$ and $G_{T}$.

The influence of the Coulomb interaction on the thermopower is analyzed in Fig. 4 as a percentage of $\left(S^{\max }-S^{\text {sat }}\right) / S^{\text {sat }}$ where $S^{\max }$ is the maximum value of the thermopower and $S^{\text {sat }}=S(t \rightarrow \infty)$. It is found that the thermopower is reduced by up to $25 \%$ for strong electron-electron interactions. This reduction is more significant at high $\Delta_{d}$. Therefore, the predicted enhancement of the thermopower up to $40 \%$ cannot be observed in strong electron-electron interactions. Indeed, an increase of correlation between electrons results in a decrease of the thermopower.

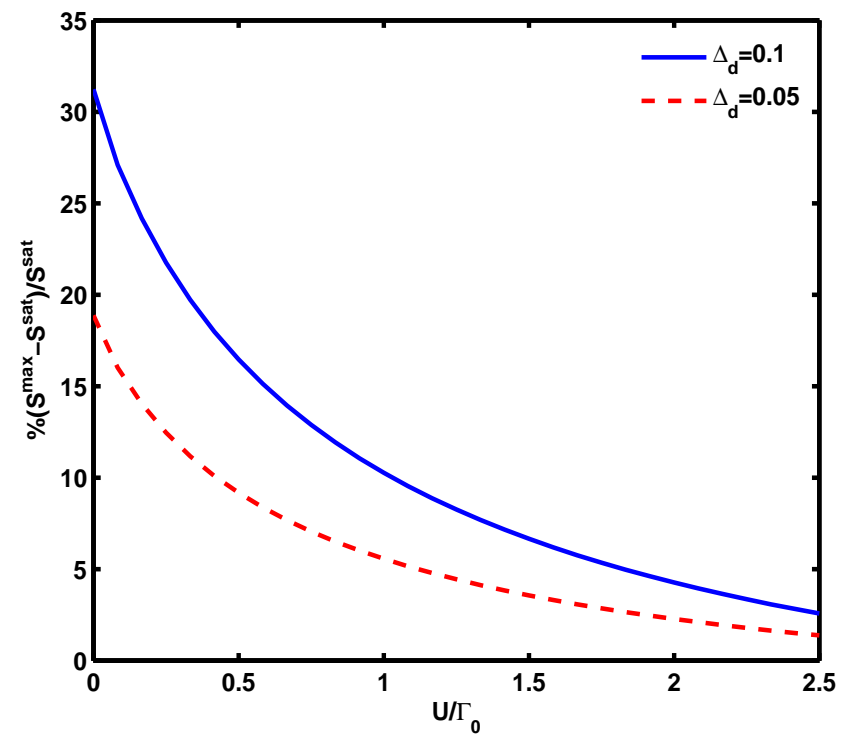

Figure 4: Percentage of $\left(S^{\max }-S(t \rightarrow \infty)\right) / S(t \rightarrow \infty)$. We set $\varepsilon_{0}=0.2$. Other parameters are the same as in Fig. 3 .

\section{Conclusion}

In this paper, we analyze the time-dependent Seebeck coefficient through an interacting quantum dot subject to a magnetic field. The formal expression of the thermopower is obtained using the nonequilibrium Green's function formalism. The influence of the magnetic field on the displacement and heat currents 
is examined, and it is observed that the current of the left and right leads are different from each other in the response to the magnetic field. Spin-dependent beats in the heat current are also observed. We find that the thermopower is

reduced by up to $25 \%$ under certain conditions. The behavior of the electrical and thermal conductances in the response to the time-dependent gate voltage are also examined.

\section{References}

[1] B. Kubala, J. König, J. Pekola, Phys. Rev. Lett. 100, 066801 (2008)

[2] Y. Dubi, M. Di Ventra, Rev. Mod. Phys. 83, 131 (2011)

[3] C. W. J. Beenakker, A. A. M. Starling, Phys. Rev. B 46, 96679676 (1992)

[4] M. Galperin, A. Nitzan, M. A. Ratner, Phys. Rev. B 75, 155312 (2007)

[5] J. Koch, F. von Oppen, Y. Oreg, E. Sela, Phys. Rev. B 70, 195107 (2004)

[6] Y. Dubi, M. Di Ventra, Phys. Rev. B 79, 081302 (2009)

[7] B. C. Hsu, Y. S. Liu, S. H. Lin, Y. C. Chen, Phys. Rev. B 83, 041404 (2011)

[8] L. G. C. Rego, G. Kirczenow, Phys. Rev. Lett. 81, 232 (1998)

[9] J. S. Wang,. J. Wang, N. Zeng, Phys. Rev. B 74, 033408 (2006)

[10] Y. Dubi, M. Di Ventra, Nano Lett. 9(1), 97 (2009)

[11] J. K. Freericks, V. Zlatic, A. M. Shvaika, Phys. Rev. B 75, 035133 (2007)

[12] M. Krawiec, K. I. Wysokinsk, Phys. Rev. B 73, 075307 (2006)

[13] M. R. Peterson, S. Mukerjee, B. S. Shastry, J. O. Haerter, Phys. Rev. B 76, $125110(2007)$

[14] K. Uchida, J. Xiao, H. Adachi, J. Ohe, S. Takahashi, J. Ieda, T. Ota, Y. Kajiwara, H. Umezawa, H. Kawai, G. E. W. Bauer, S. Maekawa, E. Saitoh, Nat. Mater. 9, 894 (2010)

[15] C. M. Jaworski, J. Yang, S. Mack, D. D. Awschalom, J. P. Heremans, R. C. Myers, Nat. Mater. 9, 898 (2010)

[16] G. Zeng, J. M. O. Zide, W. Kim, J. E. Bowers, A. C. Gossard, Z. Bian, Y. Zhang, A. Shakouri, S. L. Singer, A. Majumdar, J. Appl. Phys. 101, 034502 (2007)

[17] K. P. Pernstich, B. Rössner, B. Batlogg, Nat. mater. 7, 321 (2008)

[18] A. Tan, S. Sadat, P. Reddy, Appl. Phys. Lett. 96, 013110 (2010) 
[19] A. Crépieux, F. Simkovic, B. Cambon, F. Michelini, Phys. Rev. B 83, 153417 (2011)

[20] A. P. Jauho, N. S. Wingreen, Y. Meir, Phys. Rev. B 50, 5528 (1994)

[21] Q. F. Sun, T. H. Lin, J. Phys.: Condens. Matter 9, 4875 (1997)

[22] F. M. Souza, Phys. Rev. B 76, 205315 (2007)

[23] G. Platero, R. Aguado, Phys. Rep. 395, 1 (2004)

[24] F. M. Souza, S. A. Leão, R. M. Gester, A. P. Jauho, Phys. Rev. B 76, $125318(2007)$

[25] E. Perfetto, G. Stefanucci, M. Cini, Phys. Rev. B 78, 155301 (2008)

[26] C. Meyer, J. M. Elzerman, L. P. Kouwenhoven, Nano Lett. 7, 295 (2007)

[27] W. T. Lai, D. M. T. Kuo, P. W Li, Physica E 41, 886 (2009)

[28] L. V. Keldysh, Zh. Eksp. Teor. Fiz. 47, 1515 (1964)

[29] H. Haug, A.P. Jauho, Quantum Kinetics in Transport and Optics of Semiconductors (Springer, Heidelberg, 1996)

[30] For deriving Eq. 5, We have used decoupling approximation introduced in Ref. [21].

[31] D. C. Langreth, in Linear and Nonlinear Electron Transport in Solids, Vol. 17 of Nato Advanced Study Institute Series B: Physics, ed. by J. T. Devreese, V. E. Van Doren (Plenum, New York, 1976)

[32] For generic values of $\Gamma_{0}$, see for example, D. G. Gordon, H. Shtrikman, D. Mahalu, D. A. Magder, U. Meirav, M. A. Kastner, Nature 391, 156 (1998)

[33] E. C. Cuansing, J. S. Wang, Phys. Rev. B 81, 052302 (2010)

[34] For deriving a relation for $G_{V}$ and $G_{T}$, we assume that $\mu_{\mathrm{L}}=E_{f}+\Delta V$, $T_{\mathrm{L}}=T+\Delta T, \mu_{\mathrm{R}}=E_{f}$ and $T_{\mathrm{R}}=T$. Therefore, Fermi functions of leads can be written as $f_{\mathrm{L}}=f+\Delta V f^{\prime}-\Delta T / T f^{\prime}$ and $f_{\mathrm{R}}=f$. 